\title{
ALGUMAS DIATOMÁCEAS ENCONTRADAS SÔBRE ALGAS SUPERIORES
}

(Recebido em 15/9/1960)

Miryam B. Kutner *

As algas superiores são importantes sob vários aspectos e também pelo fato de constituirem um ótimo substrato para diatomáceas. Vivendo nos mais diversos habitats apresentam sôbre seus talos uma flora diatomológica variada, de grande interêsse para a sistemática.

Algumas das diatomáceas encontradas sôbre essas algas superiores são verdadeiramente epífitas, sendo ali o seu habitat permanente. Já outras são planctônicas e se depositam sôbre seus talos apenas trazidas pelas águas.

O material por nós estudado compõe-se de algas do gênero Centroceras sp., proveniente de Itanhaém, Ectocarpus sp. e Caulerpa racemosa de Ubatuba.

Êsse material foi submetido à oxidação por meio de permanganato de potássio a $10 \%$ e água oxigenada, até ficar completamente desprovido de matéria orgânica. Em seguida foram feitas preparações com Hyrax.

Algumas das espécies por nós estudadas foram desenhadas com o auxílio de uma câmara clara e outras fotografadas com câmara Leica.

\section{AGRADECIMENTOS}

Agradecemos ao Prof. F. C. Müller-Melchers, que nos iniciou nc estudo das diatomáceas. Ao Sr. C. Teixeira pela orientação na pesquisa dêste trabalho. Ao Sr. J. de P. Carvalho e Dra. M. Vannucci pelas valiosas sugestões e revisão do manuscrito. Ao Conselho Nacional de Pesquisas pela bôlsa concedida.

\section{RELAÇÃO DAS ESPÉCIES}

Damos a seguir uma lista que indica a composição e abundância relativa das espécies. Nela usamos os seguintes símbolos:

* Bolsista do Conselho Nacional de Pesquisas, Rio de Janeiro. Publ. n. ${ }^{\circ} 146$ do Inst. Ocean. USP. 


$$
\begin{aligned}
\mathrm{x} & =\text { muito raro } \\
\mathrm{r} & =\text { raro } \\
\mathrm{p} & =\text { pouco abundante } \\
\mathrm{a} & =\text { abundante } \\
\mathrm{m} & =\text { muito abundante }
\end{aligned}
$$

\section{ESPÉCIES}

LOCAIS

Ubatuba Itanhaém

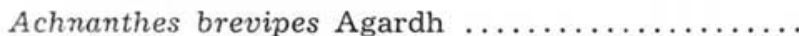

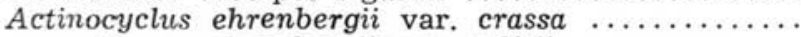

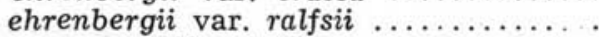

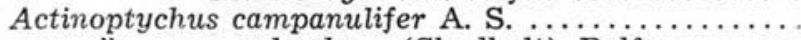

$"$ splendens (Shadbolt) Ralfs. ...........

,

undulatus (Bailey) Ralfs.

vulgaris Schumann ................

Amphora costata W. Sm.

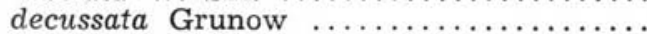

" rombica $\mathrm{Cl}$. var. intermedia Cleve .........

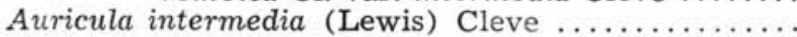

Biddulphia aurita Bréb.

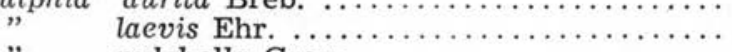

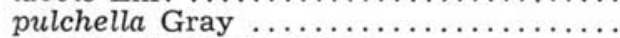

,

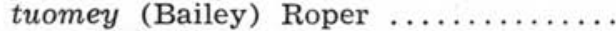

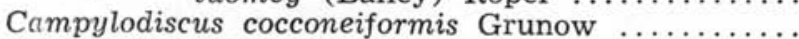

Cerataulus smithii Ralfs. turgidus Ehr.

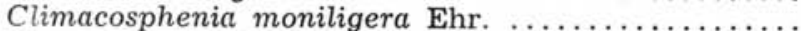

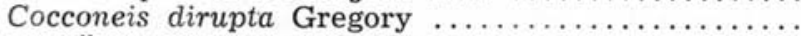

$" \quad \begin{aligned} & \text { quarnerensis Grunow } \ldots \ldots \ldots \ldots \ldots \ldots \ldots \\ & \text { pellucida Grunow } \ldots \ldots \ldots \ldots \ldots\end{aligned}$

, pinnata Gregory

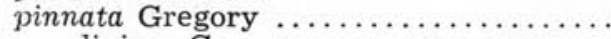

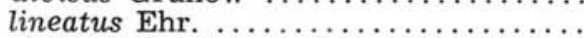

oculus-iridis Ehr.

Cyclotella stylorum Brightw.

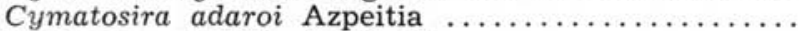

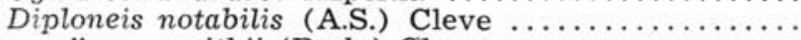

$", \quad \begin{aligned} & \text { smithii (Breb.) Cleve } \ldots \ldots \ldots \ldots \ldots \ldots \ldots \\ & , \quad \text { vacillans (A.S.) Cleve } \ldots \ldots \ldots \ldots \ldots\end{aligned}$

, weissflogii

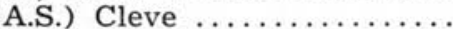

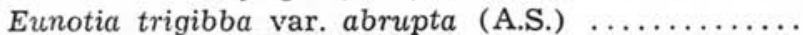

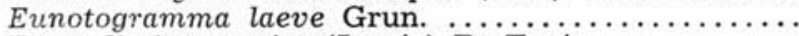

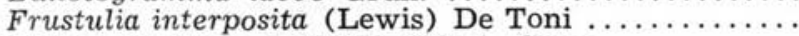

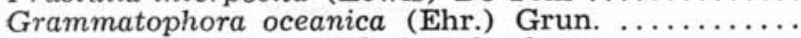

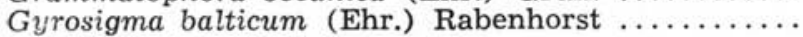

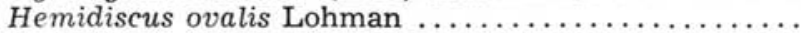

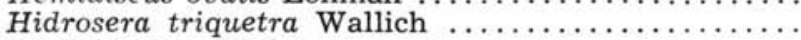

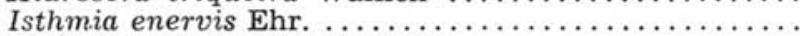

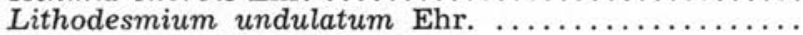

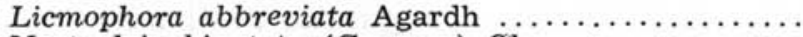

\begin{tabular}{|c|c|}
\hline $\mathbf{r}$ & - \\
\hline $\mathrm{r}$ & $\mathrm{r}$ \\
\hline $\mathbf{r}$ & $\mathbf{r}$ \\
\hline $\mathrm{p}$ & $\mathbf{r}$ \\
\hline$x$ & $\mathbf{x}$ \\
\hline$x$ & $\mathbf{x}$ \\
\hline- & $\mathrm{x}$ \\
\hline $\mathrm{p}$ & - \\
\hline $\mathrm{x}$ & - \\
\hline $\mathrm{p}$ & - \\
\hline $\mathrm{p}$ & - \\
\hline $\mathrm{r}$ & - \\
\hline- & $x$ \\
\hline - & $\mathbf{r}$ \\
\hline - & $\mathrm{r}$ \\
\hline $\bar{r}$ & $\begin{array}{l}x \\
x\end{array}$ \\
\hline $\mathrm{r}$ & - \\
\hline $\mathbf{r}$ & $\mathrm{r}$ \\
\hline $\mathrm{x}$ & - \\
\hline$x$ & - \\
\hline $\mathrm{x}$ & $\mathbf{x}$ \\
\hline $\mathbf{x}$ & - \\
\hline $\mathbf{r}$ & - \\
\hline $\mathrm{p}$ & $\mathrm{r}$ \\
\hline- & $\mathrm{r}$ \\
\hline $\mathrm{p}$ & $\mathbf{r}$ \\
\hline $\bar{x}$ & $\underline{\mathrm{r}}$ \\
\hline a & - \\
\hline $\mathrm{x}$ & - \\
\hline $\mathbf{r}$ & $\mathbf{x}$ \\
\hline - & $\mathbf{x}$ \\
\hline$x$ & $\mathbf{x}$ \\
\hline $\mathrm{r}$ & - \\
\hline p & $\mathbf{r}$ \\
\hline a & $\mathbf{r}$ \\
\hline $\mathbf{r}$ & - \\
\hline $\mathbf{r}$ & - \\
\hline $\mathbf{x}$ & - \\
\hline 二 & $\mathbf{r}$ \\
\hline $\bar{a}$ & $\mathrm{~m}$ \\
\hline $\mathrm{m}$ & - \\
\hline $\mathbf{r}$ & - \\
\hline $\mathbf{r}$ & - \\
\hline
\end{tabular}

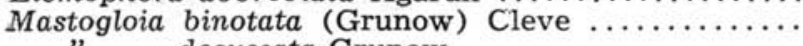

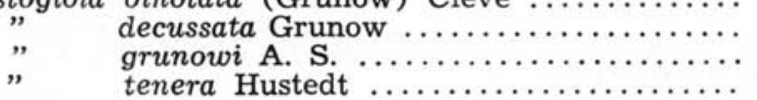
decussata Grunow $\ldots \ldots \ldots \ldots \ldots \ldots \ldots \ldots \ldots$ tenera Hustedt 


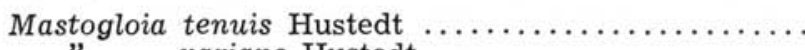

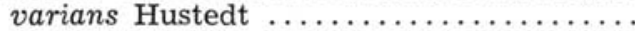

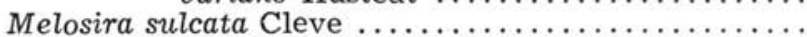

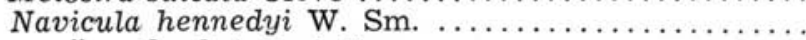

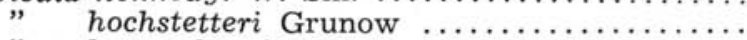

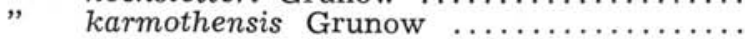

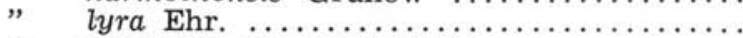

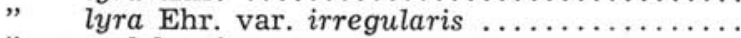

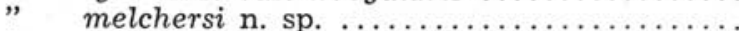

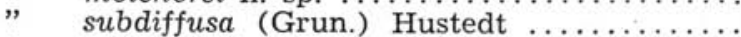

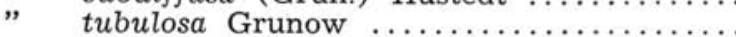

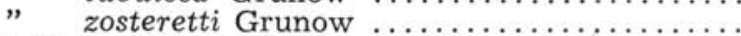

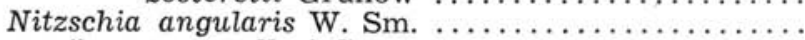

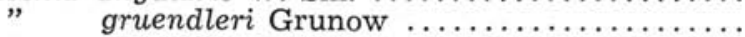

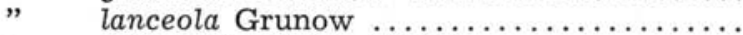

" longissima Breb. typica ..............

" marginulata var. subconstricta Grunow ..

" panduriformis Grunow ................

" panduriformis var. minor Grunow .......

,

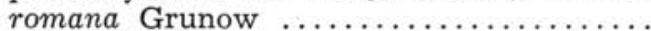

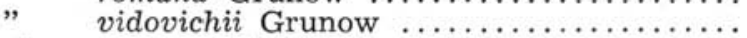

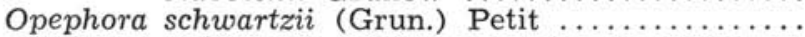

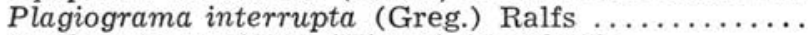

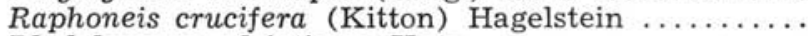

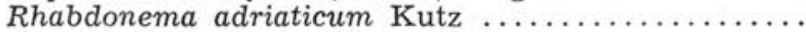

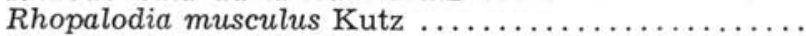

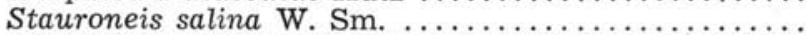

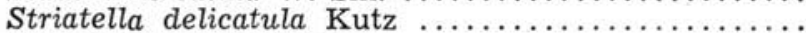

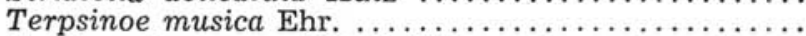

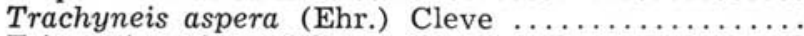

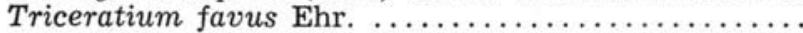

Triceratium reticulum Ehr.

Tropidoneis maxima var. sub-alata Cleve ............

\begin{tabular}{ll}
$\mathrm{a}$ & - \\
$\mathrm{x}$ & $\mathrm{r}$ \\
$\mathrm{a}$ & - \\
$\mathrm{r}$ & - \\
$\mathrm{r}$ & - \\
$\mathrm{r}$ & - \\
$\mathrm{m}$ & - \\
$\mathrm{p}$ & - \\
$\mathrm{x}$ & - \\
$\mathrm{a}$ & - \\
\hline $\mathrm{x}$ & - \\
$\mathrm{r}$ & - \\
$\mathrm{r}$ & - \\
$\mathrm{p}$ & $\mathrm{x}$ \\
$\mathrm{r}$ & - \\
\hline $\mathrm{r}$ & - \\
\hline $\mathrm{r}$ & - \\
$\mathrm{r}$ & $\mathrm{x}$ \\
$\mathrm{p}$ & - \\
$\mathrm{x}$ & - \\
\hline $\mathrm{a}$ & - \\
$\mathrm{a}$ & $\mathrm{x}$ \\
\hline $\mathrm{x}$ & $\mathrm{x}$ \\
$\mathrm{r}$ & $\mathrm{x}$ \\
$\mathrm{x}$ & - \\
$\mathrm{a}$ & - \\
\hline
\end{tabular}

Das setenta e nove espécies acima, as seguintes são assinaladas pela primeira vez nas costas brasileiras: Auricula intermedia (Lewis) Cleve, Cocconeis quarnerensis Grunow, Cocconeis pinnata Gregory, Frustulia interposita (Lewis) De Toni, Mastogloia grunowi A. Schmidt, Mastogloia tenera Hustedt, Mastogloia tenuis Hustedt, Mastogloia varians Hustedt, Nitzschia lanceola Grunow.

O gênero Auricula também é novo para o Brasil.

\section{SISTEMÁTICA}

\section{Gên. Auricula Castracanei}

Frústulas largas, arredondadas. Zona pleural complexa. Valvas reniformes ou hemisféricas, com carenas dirigidas para o mesmo lado. Rafe biarqueada. 


\section{$-6-$ \\ Auricula intermedia (Lewis) Cleve}

(Est. I, Fig. 1)

Auricula intermedia Peragallo \& Peragallo 1897-1908, est. 42, figs. 12-13. Auricula intermedia Cleve-Euler 1952, p. 30, fig. 1395.

Valvas reniformes, carenas fortemente recurvadas na parte mediana, com 5 pontos em $10 \mu$. Apresenta 50 a $60 \mu$ de comprimento, 15 a $18 \mu$ de largura, 16 a 18 estrias em $10 \mu$.

Distribuição gEOGRÁfica - Nápoles (Itália), Villefranche (França), Mar Adriático e mares quentes.

OCORRÊNCIA - Ubatuba.

\section{Cocconeis quarnerensis Grunow}

(Est. I, Fig. 2)

Cocconeis quarnerensis De Toni 1891-1894, p. 451.

Cocconeis quarnerensis Peragallo \& Peragallo 1897-1908, est. 2, figs. 7-8.

Cocconeis quarnerensis Schmidt 1874-1944, est. 192, figs. 20-24.

Cocconeis quarnerensis Hustedt 1927-1937, p. 360, fig. 814.

Cocconeis quarnerensis Frenguelli 1938, est. 1, figs. 14-15.

Cocconeis quarnerensis Cleve-Euler 1953, p. 13, fig. 503.

Valvas elípticas. Valva sem rafe com estrias radiais curtas e campo mediano largo com forma de lança, sôbre a qual se reconhecem sinais fracos de estrias. Pseudo-rafe estreita em forma de lança. Valva com rafe tem área axial muito estreita. Área central

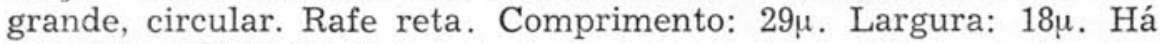
9 estrias radiais em $10 \mu$.

Distribuição geOgráfica - Costas européias. Relativamente freqüente no Mediterrâneo, mais rara nas costas setentrionais da Europa. Bahia de San Blas (Argentina).

OCORRÊNCIA - Ubatuba.

\section{Cocconeis pinnata Gregory}

(Est. I, Fig. 4)

Cocconeis pinnata Peragallo \& Peragallo 1897-1908, est. 2, figs. 11-14.

Cocconeis pinnata Schmidt 1874-1944, est. 189, figs. 1-5.

Cocconeis pinnata Hustedt 1927-1937, p. 330, fig. 783.

Valvas elípticas. Ambas estruturalmente iguais. Há de 4 a 5 estrias transapicais em $10 \mu$, que consistem de finos pontos em fileiras 
duplas, transformando-se freqüentemente em fileira de pontos simples para o lado interno. A pseudo-rafe e a área axial da valva com rafe são em forma de lança. A rafe é reta, muito delicada. Comprimento: $31 \mu$. Largura: $22 \mu$.

Distribuição GEOGRÁficA - Forma litorânea. Nas costas européias, do Mediterrâneo ao Oceano Glacial Ártico.

OCORRÊNCIA - Ubatuba.

Frustulia interposita (Lewis) De Toni

(Est. I, Fig. 5)

Frustulia interposita De Toni 1891-1894, p. 278.

Frustulia interposita Schmidt 1874-1944, est. 369, fig. 10.

Valvas elíptico-lanceoladas, rafe grossa, dupla. Estrias transversais paralelas, cêrca de $27 \mathrm{em} 10 \mu$. Estrias longitudinais onduladas, tênues, cêrca de 21 em $10 \mu$. Comprimento: $128 \mu$.

Distribuição GEOGRÁfiCA - Segundo De Toni ocorre em águas estagnadas salobras.

OCORRÊNCIA - Ubatuba.

\section{Mastogloia grunowi A. Schmidt}

(Est. II, Fig. 2)

Mastogloia grunowi Peragallo \& Peragallo 1897-1908, est. 6, fig. 30 .

Mastogloia grunowi Schmidt 1874-1944, est. 186, figs. 5-6.

Mastogloia grunowi Hustedt 1927-1937, p. 555, fig. 988.

Mastogloia grunowi Hustedt 1955, p. 20.

Valvas elíptico-lanceoladas. Áreas muito estreitas. Parede da valva com estrias transapicais radiais cruzadas por estrias longitudinais difíceis de ser vistas. De ambos os lados da linha média as estrias são interrompidas por um sulco linear mais apagado. Há 4 câmaras em $10 \mu$ que diminuem em tamanho do centro para as extremidades. Margem interna convexa. Comprimento: 35 $\mu$. Largura: $14 \mu$. Há 30 estrias transapicais em $10 \mu$.

Distribuição GEOGRÁFICA - Nas regiões costeiras dos mares quentes e no Mediterrâneo.

OCORRÊNCIA - Ubatuba. 


\section{Mastogloia tenera Hustedt \\ (Est. II, Fig. 3)}

Mastogloia tenera Hustedt 1927-1937, p. 494, fig. 919.

Valvas lanceoladas, muito ponteagudas. Rafe reta, áreas estreitas. A parede da valva com três sistemas de estrias se cruzando. Estrias transapicais mais fortes que as outras, 26 em $10 \mu$. Câmaras estreitas, ao longo de tôda a margem da valva. Seis em 10u. Margem interna convexa. Comprimento: $30 \mu$. Largura: $10 \mu$.

DistribuiçÃo GEOGRÁFICA - Nas costas da região indomalaia e nos Mares do Sul.

OCORRÊNCIA - Ubatuba.

\section{Mastogloia tenuis Hustedt \\ (Est. II, Fig. 4)}

Mastogloia tenuis Hustedt 1927-1937, p. 570, fig. 1004.

Valvas lanceoladas. Rafe reta. Áreas estreitas limitadas por duas estrias longitudinais mais fortemente silicificadas. Estrias transapicais: 34 em 10 $\mu$. Há 4 câmaras em cada margem da valva, sendo duas centrais maiores e duas terminais menores. Comprimento: $37 \mu$ (um pouco maior do que o assinalado por Hustedt). Largura: $10 \mu$.

DistribuiçÃo GEOGRÁficA - Em Miang Besar (Bornéo) porém muito rara.

OCORRÊNCIA - Ubatuba.

\section{Mastogloia varians Hustedt}

(Est. II, Fig. 5)

Mastogloia varians Hustedt 1927-1937, p. 486, fig. 909.

Valvas elípticas com extremidades rostradas. Rafe reta. Áreas axial e central muito estreitas. Aréolas em três sistemas de estrias se cruzando, mas nem todos visíveis devido ao pequeno tamanho da valva e à sua estrutura grosseira. Há 4 câmaras em $10 \mu$, cada com $1 \mu$ de largura, mais ou menos. Margem interna convexa. Câmaras terminais algo maiores que as demais. Estrias transapicais: 22 em $10 \mu$. Comprimento: $21 \mu$. Largura: $8 \mu$.

DISTRIBUIÇÃo GEOGRÁFICA - Na região costeira indomalaia e asiática oriental.

OCORRÊNCIA - Ubatuba. 
 \\ Nitzschia lanceola Grunow \\ (Est. I, Fig. 3)
}

Nitzschia lanceola De Toni 1891-1894, p. 497.

Nitzschia lanceola Van Heurck 1880-1885, est. 57, fig. 6.

Nitzschia lanceola Schmidt 1874-1944, est. 331, fig. 16.

Nitzschia lanceola Hustedt 1955, p. 44, est. 15, fig. 23.

Valvas lanceoladas com extremidades agudas. Estrias transversais com pontos delicados. Comprimento: $45 \mu$. Largura: $10 \mu$. Apresenta 9 estrias em $10 \mu$.

Distribuição GEOGRÁFICA - Freqüente nos mares quentes.

OCORRÊNCIA - Ubatuba.

\title{
Gên. Navicula Bory
}

Navicula melchersi $\mathrm{n}$. sp.

(Est. II, Fig. 1)

Valvas lanceoladas com terminações agudas. Rafe reta, ligeiramente curva nas extremidades. Área axial fortemente assimétrica. Área central pequena, um pouco dilatada transapicalmente. Estrias transapicais: 7 a $8 \mathrm{em} 10 \mu$. As estrias são "lineadas". Há 24 a 26 "lineolae" em 10 $\mu$. Comprimento: 40 a $91 \mu$. Largura: 9 a $15 \mu$.

Navicula melchersi assemelha-se a Navicula longa (Greg) Ralfs var. irregularis Hustedt e a Navicula pennata A. S. Difere delas porém por algumas características. Navicula longa var. irregularis tem menor número de estrias transapicais e de "lineolae". O mesmo acontece com Navicula pennata que ainda tem maior área central.

Navicula melchersi foi encontrada sôbre Caulerpa racemosa e Ectocarpus sp. da região de Ubatuba.

A espécie é dedicada ao Prof. F. C. Müller-Melchers do Uruguai.

\section{CONCLUSÕES}

Depreende-se da relação das espécies que a flora de diatomáceas encontrada sôbre as algas superiores de Itanhaém difere daquela das algas de Ubatuba, tanto qualitativa como quantitativamente. Vários são os determinantes dêste fato:

1 - A qualidade do substrato, ou seja, as algas que suportam as. diatomáceas são de gêneros e espécies diferentes.

2 - A diversidade dos ambientes. Em Itanhaém, por causa da arrebentação local, há uma constante renovação de água, que pela sua turbulência remove a maior parte do substrato, só permanecendo 
as diatomáceas que possuem determinados meios de adesão. Decorre dêsse fato, uma grande homogeneidade das amostras estudadas dêsse local, onde há uma ocorrência de quase $90 \%$ de diatomáceas do gênero Licmophora. Êste gênero é tìpicamente epífita pois possui pedúnculo com o qual se mantém fortemente preso ao talo da alga que lhe serve de suporte, resistindo portanto ao processo de lavagem mecânica. Isto não se dá com a maioria das demais diatomáceas aqui registradas, que ocorrem assim em pequenas quantidades.

O material de Ubatuba provém de águas mais calmas, inclusive de poças sôbre rochas, o que permite a existência de maior variedade de espécies.

\section{S U M M A R Y}

A list of diatoms found on the higher algae Centroceras sp., collected at Itanhaém, State of São Paulo, Brazil, and Ectocarpus sp. and Caulerpa racemosa from Ubatuba, also in the State of São Paulo, Brazil, is given in this paper.

Only on species Licmophora abbreviata Agardh is typically epiphitic. Nine different species and the genus Auricula, are here recorded for the first time for Brazil. A new species Navicula melchersi is described.

The difference of the surrounding conditions, calm waters at Ubatuba and breakers at Itanhaém, probably accounts for the species composition of the communities from the two places.

\section{B I B L I O G R A F I A}

Cleve-Euler, A.

1952. Die Diatomeen von Schweden und Finnland. Kungl. Svenska. Vetensk. Handl. Ser. IV, vol. 3, n. ${ }^{\circ}$ 3, p.3-153, figs. 1318-1583.

1953. Die Diatomeen von Schweden und Finnland. Kungl. Svenska Vetensk. Handl. Ser. IV, vol. 4, n. ${ }^{\circ}$ 5, p. 3-255, figs. 484-970.

DE TONI, J. B.

1891-1894. Sylloge Algarum, omnium hucusque cognitarum. Bacillarieae, 2 vols., p. 1-1556. Padova.

FRENGUELLI, J.

1938. Diatomeas de la Bahia de San Blas. Rev. Mus. La Plata, n. s., Sec. Botanica. vol. 1, p. 251-337.

HUSTEDT, F.

1927-1937. Die Kieselalgen Deutschlands, Österreichs und der Schweiz, etc. Rabenhorsts Kryptogamen-Flora, vol. 7, parte 2, fasc. 1-5.

1955. Marine litoral diatoms of Beaufort, North Carolina, Duke Univ. Mar. Stat. Bull. n. ${ }^{\circ}$ 6, p. 5-67, est. 1-16. 
Peragallo, H. \& Peragallo, M.

1897-1908. Diatomées marines de France et des districts maritimes voisins. Atlas: est. 1-137.

Schmidt, A.

1874-1944. Atlas der Diatomaceenkund. 8 vols. est. 1-416 \& 433-460. Leipzig.

VAN HEURCK, H.

1880-1885. Synopsis des diatomées de Belgique, 2 vols. Atlas: est. 1-131; Texto: p. 1-120. Anvers. 
$-12-$

ESTAMPA I

Fig. 1 - Auricula intermedia (Lewis) Cleve.

Fig. 2-Cocconeis quarnerensis Grunow.

Fig. 3 - Nitzschia lanceola Grunow.

Fig. 4 - Cocconeis pinnata Gregory.

Fig. 5 - Frustulia interposita (Lewis) De Toni. 

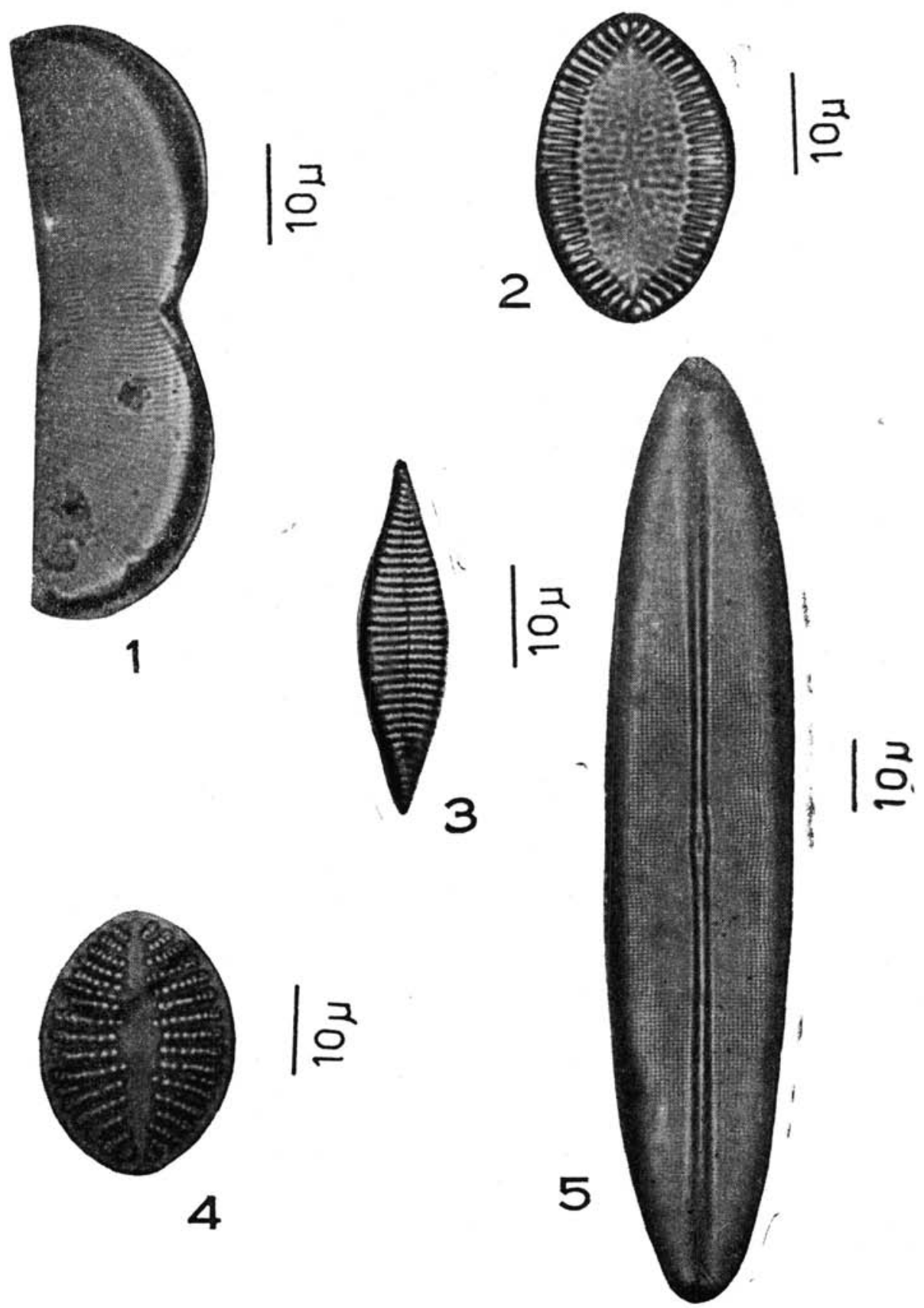
ESTAMPA II

Fig. 1 - Navicula melchersi n. sp.

Fig. 2 - Mastogloia grunowi A. Schmidt.

Fig. 3 - Mastogloia tenera Hustedt.

Fig. 4 - Mastogloia tenuis Hustedt.

Fig. 5 - Mastogloia varians Hustedt. 
KUTNER, M. B. - Algumas diatomáceas...

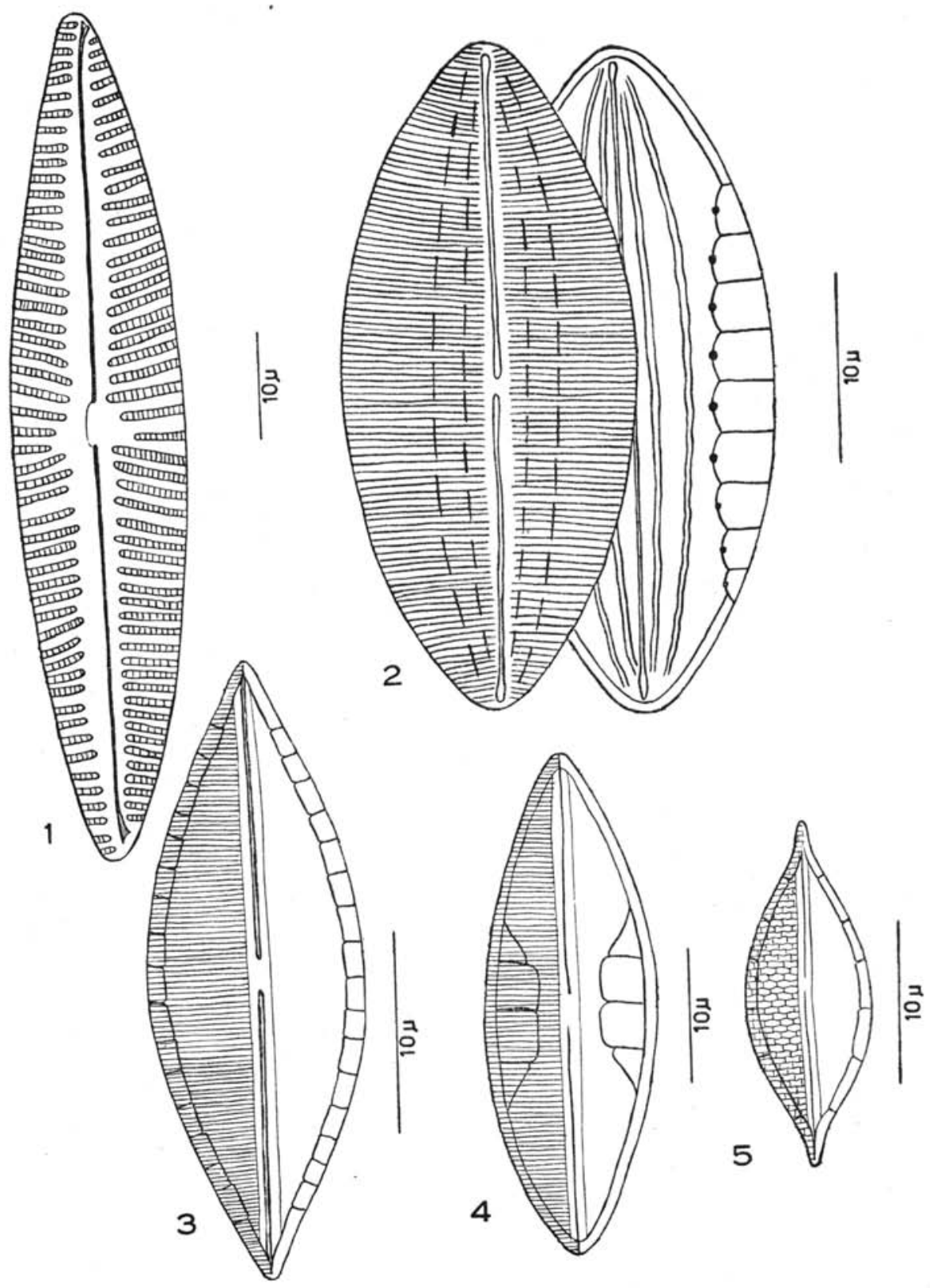

